\title{
ON EVANS' SOLUTION OF THE EQUATION $\Delta u=P u$ ON RIEMANN SURFACES
}

\author{
By Mitsuru Nakai
}

\section{Introduction.}

Let $R$ be an open Riemann surface. By a density $P(z)$ on $R$ we mean a nonnegative continuously differentiable function of local parameters $z=x+\sqrt{-1} y$ such that the expression $P(z) d x d y$ is invariant under the change of local parameters $z$. Then we can consider the elliptic partial differential equation

$$
\Delta u(z)=P(z) u(z), \quad \Delta=\frac{\partial^{2}}{\partial x^{2}}+\frac{\partial^{2}}{\partial y^{2}},
$$

which is invariantly defined on $R$. Throughout this paper, we always assume

$$
P(z) d x d y \neq 0
$$

on $R$. By a solution $u$ of $(E)$ on an open subset $D$ of $R$ we mean that $u$ is twice continuously differentiable function satisfying $(E)$ on $D .{ }^{1)}$ An Evans' solution $e(z)$ of $(E)$ on $R$ is a solution of $(E)$ on $R$ satisfying

$$
\lim _{R \ni z \rightarrow A_{\infty}} e(z)=\infty,
$$

where $A_{\infty}$ is the Alexandroff's ideal boundary point of $R$. The purpose of this paper is to give a sufficient condition for the existence of Evans' solution of $(E)$ on $R$.

Let $\left(R_{n}\right)_{n=0}^{\infty}$ be a normal exhaustion of $R$ and $\Omega_{0, n}(n>0)$ be the continuous function on $\bar{R}_{n}-R_{0}$ such that $\Omega_{0, n}$ is a solution of $(E)$ on $R_{n}-\bar{R}_{0}$ with $\Omega_{0, n}=1$ on $\partial R_{0}$ and $\Omega_{0, n}=0$ on $\partial R_{n}$. Then there exists a continuous function $\Omega_{0}$ on $R-R_{0}$ such that $\Omega_{0}$ is a solution of $(E)$ on $R-\bar{R}_{0}$ with $\Omega_{0}=1$ on $\partial R_{0}$ and

$$
\Omega_{0}(z)=\lim _{n \rightarrow \infty} \Omega_{0, n}(z)>0
$$

on $R-R_{0}$. Clearly $\Omega_{0}(z)$ does not depend on the special choice of exhaustions $\left(R_{n}\right)_{n=1}^{\infty}$.

We consider the condition

$$
\sigma=\inf _{z \in R-R_{0}} \Omega_{0}(z)>0 .
$$

It is easy to see that the condition $(\Omega)$ does not depend on the special choice of

Received September 10, 1962.

1) For fundamental properties of solutions of $(E)$, refer to the list in pp. 152-153 in [4]. 
$R_{0}$. Hence the condition $(\Omega)$ depends only on the pair $(R, P)$. The main result of this paper is the following

THeOREM 1. Suppose that the condition $(\Omega)$ is satisfied. Then the equation (E) possesses an Evans' solution of $(E)$ on $R$.

For the proof of this theorem, first we construct the extension $G(p, q)$ of the Green function on $R$ with respect to $(E)$ to the Čech compactification $R^{*}$ of $R(\S 1)$. Using this extended Green kernel $G(p, q)$, we define for each subset $K$ of $R^{*}$ a "transfinite diameter" $D(K)$ and a modified "Tchebycheff's constant" $E(K)$ of $K$ and then we prove $E(K) \geqq D(K)$ (Proposition $2, \S 2$ ). Usually in the potential theory, Fekete's relation $E(K)=D(K)$ is proved for compact sets $K$ by using symmetricity and continuity of the kernel function. But our kernel $G(p, q)$ not necessarily satisfies symmetricity and continuity. In spite of this, we can prove the half of Fekete's relation: $E(K) \geqq D(K)$. This fact may have the independent interest. Next we prove $D(\Gamma)=\infty$, where $\Gamma$ is the Čech boundary $R^{*}-R$ of $R$ (Proposition 3, $\S 3)$. After these preparations, we conclude the proof of Theorem 1 (§4).

Only in the proof of $D(\Gamma)=\infty$, we use the assumption $(\Omega)$. The converse of this is true. Namely, the following Theorem 2 holds which is proved in $\S 5$. This shows that without $(\Omega)$ the standard method due to Evans to construct Evans' solution based on the transfinite diameter with respect to the Green kernel cannot be applied.

THEOREM 2. The following conditions are mutually equivalent:

(c)

$(\Omega)$;

$$
\begin{gathered}
\inf _{z \in R} G(z, w)>0 \text { for each } w \text { in } R ; \\
D(\Gamma)=\infty .
\end{gathered}
$$

Finally we give an application of Theorem 1 to the function theory. An Evans-Selberg's potential on $R$ is a harmonic function $h(z)$ on $R$ with one negative logarithmic singularity in $R$ such that $\lim _{R \ni z \rightarrow A_{\infty}} h(z)=\infty$. Applying Theorem 1, we prove the following in $\S 6$ :

Theorem 3 (Evans-Selberg-Kuramoch $i^{2)}$ ). There exists an Evans-Selberg's potential on $R$ if and only if $R$ is of null boundary.

\section{$\S 1$. Green kernel on Čech compactification.}

Lemma 1.1. There exists a unique compact Hausdorff space $R^{*}$, called the Čech compactification of $R$, such that

(C. 1$) \quad R$ is an open subspace of $R^{*}$, or equivalently, $\Gamma=R^{*}-R$, which is called

2) See Kuramochi [2]. 
the $\check{C}$ ech boundary of $R$, is compact in $R^{*}$;

(C. 2) $R$ is dense in $R^{*}$;

(C. 3) any bounded continuous function ${ }^{3)}$ on $R$ is uniquely extended to $R^{*}$ so as to be continuous on $R^{*}$.

In fact, since $R$ is completely regular, by a theorem of $\breve{C}$ ech, ${ }^{4)}$ there exists a unique compact Hausdorff space $R^{*}$ satisfying (C. 2) and (C. 3). As $R$ is locally compact, so (C. 1 ) is satisfied for this $\left.R^{*} .{ }^{5}\right)$

Lemma 1.2. Any continuous function $f$ on $R$ is uniquely extended to $R^{*}$ so as to be continuous on $R^{*}$.

In fact, let $g(z)=\max (f(z), 0)$ and $h(z)=g(z)-f(z)$ on $R$. Then $(1+g(z))^{-1}$ and $(1+h(z))^{-1}$ are bounded continuous functions on $R$ and so continuously extended to $R^{*}$. Then the same is true for $g(z)$ and $h(z)$ and since $R$ is dense in $R^{*}$, these extensions are unique. We denote these extensions by the same notations. If $g(p)=\infty$ at some point $p$ in $R^{*}$, then there exists a neighborhood $V$ of $p$ such that $g(p)>0$ on $V$. Hence $g(z)=f(z)$ on $V \cap R$ and so $h(z)=0$ on $V \cap R$. As $V \cap R$ is dense in $V$, so $h(p)=0$. Similarly, $h(p)=\infty$ implies $g(p)=0$. Hence the expression $g(p)-h(p)$ has a definite meaning and gives a continuous extension of $f(z)=g(z)$ $-h(z)$. Again, since $R$ is dense in $R^{*}$, the extension is unique. $\quad$ Q. E.D.

Let $g(z, w)$ be the Green function on $R$ with respect to $(E)$ with its pole $w$ in $R$. It is positive, symmetric $g(z, w)=g(w, z)$ and continuous on $R \times R .^{6)}$ If we fix $z$ in $R$, then $g(z, w)$ is continuous on $R$ with respect to $w$ and so continuously extended to $R^{*}$ in a unique way. We set

$$
g(z, p)=\lim _{R \ni w \rightarrow p} g(z, w) \quad\left(p \in R^{*}\right) .
$$

Lemma 1.3. For any point $p$ in $\Gamma=R^{*}-R, g(z, p)$ is a solution of $(E)$ on $R$ and so extended continuously to $R^{*}$ in a unique way.

In fact, let $z_{0}$ be an arbitrary point in $R$ and $\varepsilon$ be an arbitrary positive number. We can find a neighborhood $U$ of $z_{0}$ with compact closure in $R$ and a positive constant $M$ such that $g\left(z_{0}, w\right) \leqq M$ if $w \notin U$. We can also find a neighborhood $V$ of $z_{0}$ such that $\bar{V} \subset U$ and $c^{-1} g\left(z_{0}, w\right) \leqq g(z, w) \leqq c g\left(z_{0}, w\right)$ for any $z$ in $V$ and $w$ in $R-U$. where $c=1+\varepsilon / M$. Hence $\left|g(z, w)-g\left(z_{0}, w\right)\right| \leqq \varepsilon$ for any $z$ in $V$ and $w$ in $R-U$. Letting $w \rightarrow p$, we get

$$
\left|g(z, p)-g\left(z_{0}, p\right)\right| \leqq \varepsilon
$$

for any $z$ in $V$. This shows that $g(z, p)$ is continuous on $R$ with respect to $z$.

3) Functions or continuous functions considered in this paper are all assumed to be $[-\infty, \infty]$-valued Bounded functions are functions whose ranges are compact in $(-\infty, \infty)$.

4) See Čech [1].

5) See p. 163 in [5].

6) See pp. 154-157 in [4]. 
Next take a countable dense subset $\left(z_{m}\right)_{m=1}^{\infty}$ of $R$. By induction, we can find sequences $\left(U_{m, n}\right)_{n=1}^{\infty}(m=1,2, \cdots)$ of neighborhoods of $p$ such that

and

$$
U_{m, n} \supset U_{m, n+1}, U_{m+1, n}, \quad \bigcap_{n=1}^{\infty}\left(R \cap U_{1, n}\right)=\phi
$$

$$
\lim _{n} \sup _{w \in U_{m, n}}\left|g\left(z_{m}, w\right)-g\left(z_{m}, p\right)\right|=0 .
$$

This is possible, since $g\left(z_{m}, w\right) \rightarrow g\left(z_{m}, p\right)$ as $w \rightarrow p$ for each $m=1,2, \cdots$. Set $V_{n}$ $=U_{n, n} \cap R$ and fix a point $w_{n}$ in $V_{n}$. Then

$$
\lim _{n} g\left(z, w_{n}\right)=g(z, p)
$$

for $z=z_{m}(m=1,2, \cdots)$. On the other hand, by Harnack type inequality, $\left(g\left(z, w_{n}\right)\right)_{n=1}^{\infty}$ is a bounded sequence of solutions on each compact subdomain except a finite number of terms. Hence by choosing a suitable subsequence, we may assume $\left(g\left(z, w_{n}\right)\right)_{n=1}^{\infty}$ converges to a solution $u(z)$ of $(E)$ on $R$. Thus $u(z)=q(z, p)$ on the dense subset $\left(z_{n}\right)_{n=1}^{\infty}$ of $R$. Since $g(z, p)$ is continuous on $R$, we conclude that $g(z, p) \equiv u(z)$ on $R$, which shows that $g(z, p)$ is a solution of $(E)$ on $R$. Q. E. D.

Definition. The Green kernel $G(p, q)$ on $R^{*}$ is defined by

$$
G(p, q)=\lim _{R \ni z \rightarrow p}\left(\lim _{R \ni w \rightarrow q} g(z, w)\right) \quad\left(p, q \in R^{*}\right) .
$$

Proposition 1. The Green kernel $G(p, q)$ on $R^{*} \times R^{*}$ possesses the following properties:

(G. 1) $G(z, w)=g(z, w)$ for $z$ and $w$ in $R$;

(G. 2) $G(z, p)=G(p, z)$ if $z$ is in $R$;

(G. 3) $G(z, p)$ is a solution of $(E)$ on $R$ except $p$;

(G. 4) $G(p, q)$ is continuous in $p \in R^{*}$ for fixed $q$ in $R^{*}$.

This is a simple consequence of hitherto considerations. Notice that we do not claim the symmetricity $G(p, q)=G(q, p)$ for $p$ and $q$ in $\Gamma$ and the continuity of $G(p, q)$ with respect to $q$ at $\Gamma$ for fixed $p$ in $\Gamma$.

From Proposition 1 and Harnack type inequality, it is easily seen that $G(z, p)$ is finitely continuous on $R \times \Gamma$ and hence continuous on $R \times R^{*}$.

\section{§2. Quantities $D(K)$ and $E(K)$.}

For each subset $K$ of $R^{*}$, we set

$$
\left(\begin{array}{l}
n \\
2
\end{array}\right) D_{n}(K)=\inf _{p_{1}, \cdots, p_{n} \in K} \sum_{i<j}^{1, \cdots, n} G\left(p_{i}, p_{j}\right) .
$$

It is easy to see that $\left(D_{n}(K)\right)_{n=1}^{\infty}$ is non-decreasing and so we can define 


$$
D(K)=\lim _{n \rightarrow \infty} D_{n}(K) .
$$

Similarly, we set

$$
n E_{n}(K)=\sup _{p_{1}, \cdots, p_{n} \in K} \inf _{p \in K} \sum_{i=1}^{n} G\left(p, p_{i}\right) .
$$

Since the sequence $\left(E_{n}(K)\right)_{n=1}^{\infty}$ satisfies

$$
(n+m) E_{n+m}(K) \geqq n E_{n}(K)+m E_{m}(K) \quad(n, m=1,2, \cdots),
$$

we can define

$$
E(K)=\lim _{n \rightarrow \infty} E_{n}(K)
$$

Proposition 2. $\quad E(K) \geqq D(K)$.

Proof. Let $n$ be an arbitrary positive integer. We set $r=1 /(n-1)$ and choose $n$ points $p_{n}, p_{n-1}, \cdots, p_{2}, p_{1}$ in $K$ satisfying

$$
\sum_{J=n-\imath+1}^{n} G\left(p_{n-\imath}, p_{j}\right) \leqq \inf _{p \in K} \sum_{j=n-\imath+1}^{n} G\left(p, p_{j}\right)+r \quad(j=1,2, \cdots, n-1) .
$$

We choose these $n$ points inductively. Let $p_{n}$ be an arbitrary point in $K$. Assume that $p_{n}, p_{n-1}, \cdots, p_{n-\imath+1}(i \leqq n-1)$ have been already chosen. Consider

$$
h(p)=\sum_{\jmath=n-\imath+1}^{n} G\left(p, p_{j}\right)
$$

Since $\inf _{p \in K} h(p) \geqq 0$, we can find a point $p_{n-\imath}$ in $K$ such that $h\left(p_{n-\imath}\right) \leqq h(p)+r$ on $K$. This is nothing but (2.1). ${ }^{7)}$

By the definition of $E_{i}(K)$, we can easily see that

$$
\inf _{p \in K} \sum_{\jmath=n-\imath+1}^{n} G\left(p, p_{j}\right) \leqq i E_{\imath}(K) .
$$

Hence by (2.1), we get

$$
\sum_{\jmath=n-\imath+1}^{n} G\left(p_{n-\imath}, p_{j}\right) \leqq i E_{\imath}(K)+r \quad(i=1,2, \cdots, n-1) .
$$

Summing up these $n-1$ inequalities, we get by the definition of $D_{n}(K)$

$$
\left(\begin{array}{c}
n \\
2
\end{array}\right) D_{n}(K) \leqq \sum_{i=1}^{n-1} i E_{i}(K)+(n-1) r
$$

or

$$
D_{n}(K) \leqq\left(\sum_{\imath=1}^{n-1} i E_{\imath}(K)\right) /\left(\begin{array}{l}
n \\
2
\end{array}\right)+1 /\left(\begin{array}{l}
n \\
2
\end{array}\right)
$$

7) Since $h(p)$ is continuous on $R^{*}$, we can choose a point $p_{n-2}$ in $K$ satisfying $h\left(p_{n-i}\right)$ $\leqq h(p)$ on $K$. Hence $r$ in (2.1) is superfluous in this case. We want to emphasize here that the above proof to show the relation $E(K) \geqq D(K)$ is valid for any kernel bounded from below. 
Since $\lim _{n} E_{n}(K)=E(K)$, it is easy to see that

$$
\lim _{n \rightarrow \infty}\left(\sum_{i=1}^{n-1} i E_{i}(K)\right) /\left(\begin{array}{l}
n \\
2
\end{array}\right)=E(K) .
$$

Hence by making $n \nearrow \infty$ in (2.2), we get $D(K) \leqq E(K)$.

\section{§3. Evaluation of $\boldsymbol{D}(\Gamma)$.}

In this section, we assume the condition $(\Omega)$ :

$$
\sigma=\inf _{R-R_{0} \ni z} \Omega_{0}(z)>0 .
$$

Under this assumption, we shall show that $D\left(I^{\prime}\right)=\infty$, where $\Gamma=R^{*}-R$.

Let $\left(R_{n}\right)_{n=0}^{\infty}$ be a normal exhaustion of $R$. Set $\Gamma_{n}=\bar{R}_{n}-\mathrm{R}_{n}$. We denote by $M\left(\Gamma_{n}\right)$ the totality of unit positive Borel measures on $\Gamma_{n}$. For each measure $\mu$ in $M\left(\Gamma_{n}\right)$, we put

$$
I(\mu)=\int G(z, w) d \mu(z) d \mu(w)
$$

Set

$$
W_{n}=\inf _{\mu \in M\left(\Gamma_{n}\right)} I(\mu)
$$

Then we have ${ }^{8)}$

Lemma 3.1. There exists a unque measure $\mu_{n}$ in $M\left(\Gamma_{n}\right)$ such that

$$
I\left(\mu_{n}\right)=W_{n}
$$

and the function $U_{n}(z)$ on $R$ defined by

$$
U_{n}(z)=\int G(z, w) d \mu_{n}(w)
$$

is the solution of $(E)$ on $R-\Gamma_{n}$ and $U_{n}(z) \leqq W_{n}$ on $R$ and $U_{n}(z)=W_{n}$ on $\Gamma_{n}$.

Let $w_{n}(z)$ be the continuous function on $R$ such that $w_{n}$ is a solution of $(E)$ in $R_{n}$ and $w_{n}=1$ on $R-R_{n}$. We set

$$
A_{n}=\int_{R} w_{n}(z) P(z) d x d y
$$

LEMMA 3.2.

$$
\lim _{n \rightarrow \infty} A_{n}=0 .
$$

Proof. The condition $\sigma>0$ implies that $R$ is of null boundary. ${ }^{9)}$ Hence $(E)$ does not possess bounded solution except the constant zero. ${ }^{10)}$ By the maximum

8) See pp. $157-165$ in [4].

9) See Theorem 1 in Ozawa [8].

10) See Ozawa [6] or Theorem 1 in Royden [9]. 


$$
\text { EVANS' SOLUTION OF } \Delta u=P u
$$

principle, $\left(w_{n}\right)$ is a monotone decreasing sequence converging to a bounded solution of $(E)$ on $R$ which must reduce to the constant zero. Hence $P(z) \geqq w_{n}(z) P(z) \backslash 0$. On the other hand, $\sigma>0$ implies

$$
\left.\int_{R} P(z) d x d y<\infty .11\right)
$$

Hence by Lebesgue's convergence theorem

$$
\lim _{n \rightarrow \infty} A_{n}=\int_{R} \lim _{n \rightarrow \infty} w_{n}(z) P(z) d x d y=0 .
$$

LEMMA 3.3.

$$
\lim _{n \rightarrow \infty} W_{n}=\infty .
$$

Proof. Let $G_{n}(z, w)$ be the Green function on $R_{n}$ with respect to $(E)$. Then $G_{n}(z, w) \nearrow G(z, w)$ on $R \times R$. By Green's formula

$$
2 \pi w_{n}(w)=\int_{\Gamma_{n}} * d_{z} G_{n}(z, w)=-\int_{R_{n}} d_{z}\left(* d_{z} G_{n}(z, w)\right)+2 \pi .
$$

Since $d_{z}\left({ }^{*} d_{z} G_{n}(z, w)\right)=A_{z} G_{n}(z, w)=P(z) G_{n}(z, w)$, we get

$$
\int_{R_{n}} G_{n}(z, w) P(z) d x d y=2 \pi\left(1-w_{n}(z)\right)
$$

Hence by making $n \nearrow \infty$, we get

$$
\int_{R} G(z, w) P(z) d x d y=2 \pi .^{12)}
$$

From this, by Fubini's theorem

Thus

$$
\int_{R} U_{n}(z) P(z) d x d y=\int_{\Gamma_{n}}\left(\int_{R} G(z, w) P(z) d x d y\right) d \mu_{n}(w)=2 \pi .
$$

$$
\int_{R} U_{n}(z) P(z) d x d y=2 \pi
$$

By the maximum principle, $U_{n}(z) \leqq W_{n} w_{n}(z)$ on $R$. From this

$$
\int_{R} U_{n}(z) P(z) d x d y \leqq W_{n} \int_{R} w_{n}(z) P(z) d x d y .
$$

Hence by (3.1), we get $2 \pi \leqq W_{n} A_{n}$ and so

$$
\liminf _{n \rightarrow \infty} W_{n} \geqq \lim _{n \rightarrow \infty} 2 \pi / A_{n}=\infty .
$$

LFMma 3.4 .

$$
D(\Gamma) \geqq \sigma^{2} W_{m} \quad(m=1,2, \cdots) .
$$

11) See Corollary 1 in Ozawa [8].

12) This relation is due to L. Myrberg [3] and Ozawa [7]. 
Proof. Let $\Omega_{m, k}(z)(k>m)$ be a continuous function on $\bar{R}_{k}-R_{m}$ such that $\Omega_{m, k}$ is the solution of $(E)$ in $R_{k}-\bar{R}_{m}$ with $\Omega_{m, k}=1$ on $\Gamma_{m}$ and $\Omega_{m, k}=0$ on $\Gamma_{k}$. Clearly $\left(\Omega_{m, k}\right)_{k=m+1}^{\infty}$ is an increasing sequence and so there exists a continuous function $\Omega_{m}$ on $R-R_{m}$ which is a solution of $(E)$ on $R-\bar{R}_{m}$ with $\Omega_{m}=1$ on $\Gamma_{m}$ and

$$
\lim _{k \rightarrow \infty} \Omega_{m, k}(z)=\Omega_{m}(z)
$$

on $R-R_{m}$. Since $\Omega_{m, k} \geqq \Omega_{0, k}$ on $R_{k}-R_{m}$ by the maximum principle, we have

$$
\Omega_{m}(z) \geqq \Omega_{0}(z)
$$

on $R-R_{m}$. Hence in particular

$$
\inf _{z \in R-R_{m}} \Omega_{m}(z) \geqq \sigma .
$$

Let $n$ be an arbitrary positive integer larger than 4 and $p_{1}, p_{2}, \cdots, p_{n}$ be in $\Gamma$. We choose $n$ points $q_{1}, q_{2}, \cdots, q_{n}$ in $\Gamma_{m}$ inductively as follows. Let

$$
h_{1}(z)=\sum_{\imath=2}^{n} G\left(z, p_{\imath}\right)
$$

and $q_{1}$ be in $\Gamma_{m}$ such that

$$
h_{1}\left(q_{1}\right)=\min _{z \in \Gamma_{m}} h_{1}(z) .
$$

Since $h_{1}(z) \geqq 0$ on $R_{k}-R_{m}$, we have by the maximum principle, $h_{1}(z) \geqq h_{1}\left(q_{1}\right) \Omega_{m, k}(z)$ for $z$ in $R_{k}-R_{m}$. Hence on $R^{*}-R_{m}$

$$
h_{1}(p) \geqq h_{1}\left(q_{1}\right) \Omega_{m}(p) .
$$

Hence in particular $h_{1}\left(p_{1}\right) \geqq h_{1}\left(q_{1}\right) \Omega_{m}\left(p_{1}\right) \geqq \sigma h_{1}\left(q_{1}\right)$ and so

$$
\sigma \sum_{i=2}^{n} G\left(q_{1}, p_{i}\right)+\sum_{i<j}^{2, \cdots, n} G\left(p_{i}, p_{j}\right) \leqq \sum_{i<j}^{1, \cdots, n} G\left(p_{i}, p_{j}\right) \equiv a .
$$

Next we choose $q_{2}, q_{3}, \cdots, q_{n-2}$ in $\Gamma_{m}$ satisfying

$$
\sigma^{2} \sum_{i<j}^{1, \cdots, k} G\left(q_{i}, q_{j}\right)+\sigma \sum_{i=1}^{k} \sum_{\jmath=k+1}^{n} G\left(q_{i}, p_{j}\right)+\sum_{i<j}^{k+1, \cdots, n} G\left(p_{i}, p_{\jmath}\right) \leqq a \quad(k=2,3, \cdots, n-2) .
$$

First let

$$
h_{2}(z)=\sum_{j=3}^{n} G\left(z, p_{j}\right)+\sigma G\left(q_{1}, z\right)
$$

and $q_{2}$ be in $\Gamma_{m}$ such that

$$
h_{2}\left(q_{2}\right)=\min _{z \in \Gamma_{m}^{\prime}} h_{2}(z) .
$$

Similarly as above, we have $h_{2}(p) \geqq h_{2}\left(q_{2}\right) \Omega_{m}(p)$ on $R^{*}-R_{m}$ and so

$$
\sum_{j=3}^{n} G\left(p_{2}, p_{j}\right)+\sigma G\left(q_{1}, p_{2}\right) \geqq \sigma \sum_{j=3}^{n} G\left(q_{2}, p_{j}\right)+\sigma^{2} G\left(q_{1}, q_{2}\right) .
$$


From this with (3.2), we get

$$
\sigma^{2} G\left(q_{1}, q_{2}\right)+\sigma \sum_{i=1}^{2} \sum_{j=3}^{n} G\left(q_{i}, p_{j}\right)+\sum_{i<j}^{3, \cdots, n} G\left(p_{i}, p_{j}\right) \leqq a .
$$

This is nothing but (3.3) for $k=2$. Next assume that $q_{2}, \cdots, q_{k}(k \leqq n-3)$ have been chosen in $\Gamma_{m}$ satisfying (3.3). Let

$$
h_{k+1}(z)=\sum_{\jmath=k+2}^{n} G\left(z, p_{\jmath}\right)+\sigma \sum_{i=1}^{k} G\left(q_{i}, z\right)
$$

and $q_{k+1}$ be in $\Gamma_{m}$ such that

$$
h_{k+1}\left(q_{k+1}\right)=\min _{z \in \Gamma_{m}} h_{k+1}(z) .
$$

Similarly as before, we have $h_{k+1}(p) \geqq h_{k+1}\left(q_{k+1}\right) \Omega_{m}(p)$ on $R^{*}-R_{m}$ and so

$$
\begin{aligned}
& \sum_{\jmath=k+2}^{n} G\left(p_{k+1}, p_{j}\right)+\sigma \sum_{i=1}^{k} G\left(q_{\imath}, p_{k+1}\right) \\
\geqq & \sigma \sum_{j=k+2}^{n} G\left(q_{k+1}, p_{j}\right)+\sigma^{2} \sum_{l=1}^{k} G\left(q_{i}, q_{k+1}\right) .
\end{aligned}
$$

From this with (3.3), we have

$$
\sigma^{2} \sum_{i<j}^{1, \cdots, k+1} G\left(q_{i}, q_{j}\right)+\sigma \sum_{i=1}^{k+1} \sum_{\jmath=k+2}^{n} G\left(q_{i}, p_{j}\right)+\sum_{i<j}^{k+2, \cdots, n} G\left(p_{i}, p_{j}\right) \leqq a .
$$

This is (3.3) for $k+1$. Thus we have constructed the system $q_{2}, \cdots, q_{n-2}$. Next let

$$
h_{n-1}(z)=G\left(z, p_{n}\right)+\sigma \sum_{i=1}^{n-2} G\left(q_{\imath}, z\right)
$$

and $q_{n-1}$ be in $\Gamma_{m}$ such that

$$
h_{n-1}\left(q_{n-1}\right)=\min _{z \in \Gamma_{m}} h_{n-1}(z) .
$$

Similarly as before, we have $h_{n-1}(p) \geqq h_{n-1}\left(q_{n-1}\right) \Omega_{m}(p)$ on $R^{*}-R_{m}$ and so

$$
G\left(p_{n-1}, p_{n}\right)+\sigma \sum_{i=1}^{n-2} G\left(q_{i}, p_{n-1}\right) \geqq \sigma G\left(q_{n-1}, p_{n}\right)+\sigma^{2} \sum_{i=1}^{n-2} G\left(q_{i}, q_{n-1}\right) .
$$

From this with (3.3) for $k=n-2$, we have

$$
\sigma^{2} \sum_{i<j}^{1, \cdots, n-1} G\left(q_{i}, q_{j}\right)+\sigma \sum_{i=1}^{n-1} G\left(q_{i}, p_{n}\right) \leqq a .
$$

Finally let

$$
h_{n}(z)=\sigma \sum_{i=1}^{n-1} G\left(q_{i}, z\right)
$$

and $q_{n}$ be in $\Gamma_{m}$ such that 


$$
h_{n}\left(q_{n}\right)=\min _{z \in \Gamma_{m}} h_{n}(z) .
$$

Similarly as before, we have $h_{n}(p) \geqq h_{n}\left(q_{n}\right) \Omega_{m}(p)$ on $R^{*}-R_{m}$ and so

$$
\sigma \sum_{\imath=1}^{n-1} G\left(q_{i}, p_{n}\right) \geqq \sigma^{2} \sum_{l=1}^{n-1} G\left(q_{i}, q_{n}\right) .
$$

From this with (3.4), we get

$$
\sigma^{2} \sum_{i<j}^{1, \cdots, n} G\left(q_{i}, q_{j}\right) \leqq a=\sum_{i<j}^{1, \cdots, n} G\left(p_{i}, p_{j}\right) .
$$

From this inequality, we get by the definition of $D_{n}\left(\Gamma_{m}\right)$

$$
\sigma^{2}\left(\begin{array}{l}
n \\
2
\end{array}\right) D_{n}\left(\Gamma_{m}\right) \leqq \sum_{i<j}^{1, \cdots, n} G\left(p_{\imath}, p_{j}\right) .
$$

Since $p_{1}, \cdots, p_{n}$ are arbitrary in $\Gamma$, we get

$$
\sigma^{2}\left(\begin{array}{l}
n \\
2
\end{array}\right) D_{n}\left(I_{m}^{\prime}\right) \leqq\left(\begin{array}{c}
n \\
2
\end{array}\right) D_{n}\left(I^{\prime}\right) \quad \text { or } \quad D_{n}(\Gamma) \geqq \sigma^{2} D_{n}\left(\Gamma_{m}^{\prime}\right) .
$$

Hence by making $n \nearrow \infty$, we get

$$
D(\Gamma) \geqq \sigma^{2} D\left(\Gamma_{m}\right) .
$$

Now let $q_{2}^{(n)}, \cdots, q_{n}^{(n)}$ be in $\Gamma_{m}$ with

$$
\left(\begin{array}{l}
n \\
2
\end{array}\right) D_{n}\left(\Gamma_{m}\right)+1 \geqq \sum_{i<j}^{1, \cdots, n} G\left(q_{i}^{(n)}, q_{j}^{(n)}\right)
$$

and let $\mu_{n}$ be in $M\left(\Gamma_{m}\right)$ with $\mu_{n}\left(q_{\imath}^{(n)}\right)=1 / n(i=1, \cdots, n)$. Since $M\left(\Gamma_{m}\right)$ is vaguely compact, ${ }^{13)}$ there exists a subsequence $\left(\mu_{n^{\prime}}\right)$ of $\left(\mu_{n}\right)$ and a measure $\mu$ in $M\left(I_{m}^{\prime}\right)$ with $\mu=\lim _{n^{\prime} \rightarrow \infty} \mu_{n^{\prime}}$ (vaguely). Let $c$ be an arbitrary positive number. From (3.6)

Hence

$$
\begin{aligned}
\left(\begin{array}{c}
n^{\prime} \\
2
\end{array}\right) D_{n^{\prime}}\left(\Gamma_{m}\right)+1 & \geqq \sum_{\imath<j}^{1, \cdot n^{\prime}} \min \left(c, G\left(q_{\imath}^{\left(n^{\prime}\right)}, q_{j}^{\left(n^{\prime}\right)}\right)\right) \\
& =\frac{n^{\prime 2}}{2} \int \min (c, G(z, w)) d \mu_{n^{\prime}}(z) d \mu_{n^{\prime}}(w)-\frac{n^{\prime} c}{2} .
\end{aligned}
$$

$$
D\left(\Gamma_{m}\right)+1 /\left(\begin{array}{c}
n^{\prime} \\
2
\end{array}\right) \geqq \int \min (c, G(z, w)) d \mu_{n^{\prime}}(z) d \mu_{n^{\prime}}(w)-\frac{c}{n^{\prime}} .
$$

As $\mu_{n^{\prime}} \times \mu_{n^{\prime}}$ converges to $\mu \times \mu$ vaguely and $\min (c, G(z, w))$ is continuous on $\Gamma_{m} \times \Gamma_{m}$, so by making $n^{\prime} \nearrow \infty$,

$$
D\left(\Gamma_{m}\right) \geqq \int \min (c, G(z, w)) d \mu(z) d \mu(w) .
$$

13) See Selection theorem, p. 162 in [4]. 
Making $c \nearrow \infty$, we have $D\left(\Gamma_{m}\right) \geqq I(\mu) \geqq \inf _{\nu \in M\left(\Gamma_{m}\right)} I(\nu)$, i. e.

$$
D\left(\Gamma_{m}\right) \geqq W_{m} .
$$

From this with (3.5), we finally get

$$
D(\Gamma) \geqq \sigma^{2} W_{m} .
$$

Proposition 3.

$$
D\left(I^{\prime}\right)=\infty \text {. }
$$

This follows from Lemmas 3.3 and 3.4 .

\section{§4. Proof of Theorem 1 .}

Assume that the condition $(\Omega)$ is satisfied:

$$
\inf _{z \in R-R_{0}} \Omega_{0}(z)>0 \text {. }
$$

We have to prove the existence of an Evans' solution of $(E)$ on $R .^{14)}$

By Propositions 2 and 3 , we have $E(\Gamma)=\infty$. Since $E(\Gamma)=\lim _{n} E_{n}(\Gamma)$, we can find an increasing sequence $\left(n_{k}\right)_{k=1}^{\infty}$ of positive integers such that

$$
E_{n_{k}}(\Gamma)>2^{k} \quad(k=1,2, \cdots) .
$$

By the definition of $E_{n_{k}}(\Gamma)$, we can find $n_{k}$ points $p_{k, \imath}\left(i=1,2, \cdots, n_{k}\right)$ in $\Gamma$ such that

Then the function

$$
\inf _{p \in \Gamma} \sum_{i=1}^{n_{k}} G\left(p, p_{k, \imath}\right)>2^{k} n_{k} \text {. }
$$

$$
e_{k}(p)=2^{-k-1} n_{k}^{-1} \sum_{\imath=1}^{n_{k}} G\left(p, p_{k, \imath}\right)
$$

is continuous on $R^{*}$ and a solution of $(E)$ on $R$ and $e_{k}(p)>1 / 2$ on $\Gamma$. Thus we can find a compact set $K_{k}$ in $R$ such that

$$
e_{k}(p)>\frac{1}{2} \text { on } R^{*}-K_{k} \text { and a fortiori on } R-K_{k} \text {. }
$$

Let $z_{0}$ be a point in $R$ and $V_{0}$ be a neighborhood of $z_{0}$ with $\bar{V}_{0}$ compact in $R$. Then there exists a constant $c_{0}$ such that $G\left(z_{0}, w\right) \leqq c_{0}\left(w \in R-V_{0}\right)$. Hence $G\left(z_{0}, p\right) \leqq c_{0}$ for any $p$ in $\Gamma$. From this, $e_{k}\left(z_{0}\right) \leqq c_{0} / 2^{k+1}$. Thus the sequence $\left(\sum_{k=1}^{n} e_{k}(z)\right)_{n=1}^{\infty}$ is a monotone increasing sequence of solutions of $(E)$ on $R$ such that $\sum_{k=1}^{n} e_{k}\left(z_{0}\right) \leqq c_{0}$. Hence

$$
e(z)=\sum_{k=1}^{\infty} e_{k}(z)
$$

14) The following method of construction is the standard one originally due to Evans. The following proof is contained only for the sake of completeness. 
is a solution of $(E)$ on $R$ and $e(z) \geqq n / 2$ on $R-\bigcup_{k=1}^{n} K_{k}$. Since $\bigcup_{k=1}^{n} K_{k}$ is compact in $R$, the above inequality shows that $\lim _{R \ni z \rightarrow A_{\infty}} e(z)=\infty$. Thus the function $e(z)$ is a required Evans' solution of $(E)$ on $R$.

REMARK. The Evans' solution $e(z)$ of $(E)$ on $R$ constructed above satisfies the following condition:

$$
\int_{R} e(z) P(z) d x d y<\infty
$$

In fact, by the footnote ${ }^{12)}$

$$
\int_{R} G(z, w) P(z) d x d y=2 \pi
$$

for any $w$ in $R$. Hence by Fatou's lemma

$$
\int_{R} G(z, p) P(z) d x d y \leqq \liminf _{R \ni w \rightarrow p} \int_{R} G(z, w) P(z) d x d y=2 \pi .
$$

Thus

$$
\int_{R} e_{k}(z) P(z) d x d y=2^{-k-1} n_{k}^{-1} \sum_{i=1}^{n_{k}} \int_{R} G\left(z, p_{k, \imath}\right) P(z) d x d y \leqq 2^{-k-1} 2 \pi
$$

and so

$$
\int_{R} e(z) P(z) d x d y=\sum_{k=1}^{\infty} \int_{R} e_{k}(z) P(z) d x d y \leqq 2 \pi .
$$

It is the writer's conjecture that the condition $(\Omega)$ is equivalent to the existence of Evans' solution satisfying the condition $(*)$.

\section{$\S 5$. Proof of Theorem 2 .}

To see the equivalence of (a) and (b), we have only to show that (a) is equivalent to

$$
\inf _{z \in R} G(z, w)>0
$$

for a fixed $w$ in $R_{0}$. Let $c>1$ and satisfy $c>G(z, w)>c^{-1}$ for any $z$ in $\partial R_{0}$ and $G_{n}(z, w)$ be Green's function of $(E)$ on $R_{n}$. Then for sufficiently large $n, c>G_{n}(z, w)$ $>c^{-1}$ for $z$ in $\partial R_{0}$. Hence by the maximum principle

$$
c \Omega_{0, n}(z)>G_{n}(z, w)>c^{-1} \Omega_{0, n}(z)
$$

on $R_{n}-\bar{R}_{0}$. From this we get

$$
c \Omega_{0}(z)>G(z, w)>c^{-1} \Omega_{0}(z) .
$$

This shows that the equivalence of (a) and (b).

The implication (a) $\rightarrow$ (c) is nothing but Proposition 3 
Finally we show that the implication $(c) \rightarrow(b)$. Contrary to the assertion, assume that

$$
\inf _{z \in R} G(z, w)=0
$$

for a point $w$ in $R$. Then there exists a point $p$ in $\Gamma$ such that $G(w, p)=G(p, w)=0$ for a point $w$ in $R$ and so for every point $w$ in $R$. Thus for any $q$ in $\Gamma, G(q, p)=0$. Hence by putting $q_{1}=q_{2}=\cdots=q_{n}=p$, we get

$$
0 \leqq \inf _{p_{1}, \cdots, p_{n} \in \Gamma} \sum_{i<j}^{1, \cdots, n} G\left(p_{i}, p_{j}\right) \leqq \sum_{i<j}^{1, \cdots, n} G\left(q_{i}, q_{j}\right)=0
$$

or

$$
D_{n}(\Gamma)=0 .
$$

Thus $D(\Gamma)=\lim _{n} D_{n}(\Gamma)=0$, which is a contradiction.

Q.E.D.

From the above proof, we also get

Theorem 2'. The following conditions are mutually equivalent:

$$
\begin{gathered}
\inf _{z \in R-R_{0}} \Omega_{0}(z)=0 ; \\
\inf _{z \in R} G(z, w)=0 \text { for every } w \text { in } R ; \\
D(\Gamma)=0 .
\end{gathered}
$$

REMARK. The condition $(\Omega)$ is a sufficient but not necessary condition for the existence of Evans' solution of $(E)$ on $R$. As an example, ${ }^{15)}$ let

and

$$
R=(z ;|z|<1)
$$

$$
P(z)=\frac{4\left(1+|z|^{2}\right)}{\left(1-|z|^{2}\right)^{2}}
$$

Then $(E)$ possesses an Evans' solution

$$
e(z)=\frac{1}{1-|z|^{2}}
$$

of $(E)$ on $R$. To show that the condition $(\Omega)$ is not satisfied, let $\left(R_{n}\right)_{n=0}^{\infty}$ be the exhaustion of $R$ such that $R_{n}=(z ;|z|<1-1 /(n+2))$ and $w_{n}(z)$ be the harmonic function on $R_{n}-\bar{R}_{0}(n \geqq 1)$ with boundary value $w_{n}=1$ on $\partial R_{0}$ and $w_{n}=0$ on $\partial R_{n}$. Then by the maximum principle

$$
\Omega_{0, n}(z) \leqq w_{n}(z) .
$$

Clearly $w_{n}$ converges to the harmonic function $w$ on $R-\bar{R}_{0}$ with boundary value $w=1$ on $\partial R_{0}$ and $w=0$ on $\partial R=(z ;|z|=1)$. Hence

15) This example is due to Royden [9], p. 10. 
and so

$$
0<\Omega_{0}(z) \leqq w(z)
$$

$$
0 \leqq \inf _{R-R_{0} \ni z} \Omega_{0}(z) \leqq \inf _{R-R_{0} \ni z} w(z)=0 .
$$

It is the Prof. Ozawa's conjecture that the non-existence of non-zero bounded solution of $(E)$ on $R$ is equivalent to the existence of Evans' solution of $(E)$ on $R \cdot{ }^{16)}$

\section{$\S 6$. Proof of Theorem 3 .}

The "only if" part of Theorem 3 is easily seen and well known. So we have only to show the "if" part. Take a density $P(z)$ with $P(z) d x d y \neq 0$ on $R$ and $P(z) d x d y \equiv 0$ outside a fixed compact set in $R$. Let $\left(R_{n}\right)_{n=0}^{\infty}$ be a normal exhaustion of $R$. We assume that $P(z) d x d y \equiv 0$ on $R-R_{0}$. Then the function $\Omega_{0, n}(z)(n \geqq 1)$ is harmonic in $R_{n}-\bar{R}_{0}$ with boundary value $\Omega_{0, n}=1$ on $\partial R_{0}$ and $\Omega_{0, n}=0$ on $\partial R_{n}$. Hence $\Omega_{0}$ is the positive harmonic function on $R-\bar{R}_{0}$ with boundary value $\Omega_{0}=1$ on $\partial R_{0}$. Since $R$ is of null boundary, by well known Mori's theorem,

$$
\sigma=\inf _{z \in R-R_{0}} \Omega_{0}(z)>0,
$$

i. e. the condition $(\Omega)$ is satisfied. Hence by Theorem 1, there exists an Evans' solution $e(z)$ of $(E)$ on $R$. As

$$
\Delta e(z)=P(z) e(z)=0
$$

on $R-R_{1}$, so $e(z)$ is harmonic on $R-R_{1}$. Let $a>\sup _{z \in R_{1}} e(z)$ and $U=(z \in R ; e(z)>a)$. We can find a positive number $b$ such that

$$
b \int_{\partial U} * d e(z)=2 \pi
$$

Let the singularity function $s(z)$ on $R-\partial U$ be defined as follows:

$$
s(z)= \begin{cases}b e(z)-a b & \text { on } U, \\ -k(z, w) & \text { on } R-\bar{U},\end{cases}
$$

where $k(z, w)$ is the harmonic Green's function on $R-\bar{U}$ with its pole $w$ in $R-\bar{U}$. Let $L$ be a normal operator of Sario. ${ }^{17)}$ Consider the equation

By the definition of $s(z)$,

$$
L(h-s)=h-s .
$$

$$
\int_{\partial U+\partial(R-U)} * d s(z)=0
$$

and so the above equation has a solution $h(z)$ harmonic on $R$ which is a desired Evans-Selberg's potential on $R$ with one negative logarithmic pole at $w^{17}$ )

16) Compare this with the conjecture in the remark of $\S 4$.

17) See Sario [10]. 


\section{REFERENCES}

[1] ČECH, E., On bicompact spaces. Ann. of Math. 38 (1937), 823-844.

[2] Kuramochi, Z., Mass distributions on the ideal boundaries of abstract Riemann surfaces, I. Osaka Math. J. 8 (1956), 119-137.

[3] Myrberg, L., Über die Existenz der Greenschen Funktion der Gleichung $\Delta u$ $=c(P) u$ auf Riemannschen Flächen. Ann. Acad. Sci. Fenn. AI 170 (1954), 1-8.

[4] NAKAI, M., The space of non-negative solutions of the equation $\Delta u=p u$ on a Riemann surface. Kōdai Math. Sem. Rep. 12 (1960), 151-178.

[5] ___ Algebraic criterion on quasiconformal equivalence of Riemann surfaces. Nagoya Math. J. 16 (1960), 157-184.

[6] Ozawa, M., Classification of Riemann surfaces. Kōdai Math. Sem. Rep. 4 (1952), 63-76.

[7] - Some classes of positive solutions of $\Delta u=P u$ on Riemann surfaces, I. Ködai Math. Sem. Rep. 6 (1954), 121-126.

[8] - A set of capacity zero and the equation $\Delta u=P u$. Kōdai Math. Sem. Rep. 12 (1960), 76-81.

[9] Royden, H. L., The equation $\Delta u=P u$, and the classification of open Riemann surfaces. Ann. Acad. Sci. Fenn. AI 271 (1959), 1-26.

[10] SARIO, L., A Linear operator method on arbitrary Riemann surfaces. Trans. Amer. Math. Soc. 72 (1952), 281-295.

Mathematical Institute,

NAGOYA UNIVERSITY. 